\title{
Flow property measurement of rice flour- A Review
}

\author{
Shumaila Jan ${ }^{\mathrm{a}}$, Kulsum Jan and DC Saxena \\ Department of Food Engineering and Technology \\ Sant Longowal Institute of Engineering \& Technology, Longowal - 148 106, Punjab (INDIA)
}

\begin{abstract}
This review covers conventional and new methods used to measure flowability of rice flour. Powder flowability is inherently complex due to many physical and environmental variables that determine how it responds to being moved. Investigating flowability requires a high specification powder rheometer having exceptional sensitivity and repeatability of measurement. This study shows how rice flour can be characterized regarding flowability in relation to flow rate, compaction and other factors.
\end{abstract}

\section{Introduction}

Rice (Oryza sativa L.) is the most important cereal crop and staple food of over the world. More than 2000 million people obtain their $60-70 \%$ calories from rice and its products [1]. It is also used in large number of food products like idli, dosa, noodles, breakfast cereals, unleavened breads, snack food items, crackers, candies and baby foods etc. [2]. Flowability is defined as the ability of a powder to flow freely, in a regular and a constant way [3]. This property establishes the performance and the nature of the working processes (mixture, transport, storage) and/or applications of the powder. So, a powder with good flowability flows without assistance, whereas a cohesive powder requires a mechanical device adapted to its actuation through agitation, vibration, etc. With the increasing quantity and variety of powders/flours being produced in industry, there is a need for information about their handling and processing characteristics. During flow, individual particles predominantly slide across each other in a shearing action. However, food powder particles can also stick together and form stable structures, preventing flow due to mechanical forces. Mechanical and chemical interactions among powders depend on composition, moisture content, surface mechanical characteristics, particle history (e.g., from production to storage), particle shape and size, and the manner in which the particles geometrically interact to form stable structures. Consequently, the strength among particles can be due to mechanical interlocking, chemical bonding, and van der Waals and electrostatic forces. The forces opposing flow are friction, attraction between particles (cohesion), attraction between particles and system walls, and particle interlocking [4].

\section{Application of Rice Flour}

The rice flour is an important ingredient in both traditional and novel foods prepared across the world [5]. Some of the traditional rice flour based products in India are puttu, made by steaming rice flour and grated coconut, and appam, a cake formed by mixing rice flour and fermented coconut milk. Rice flour exhibits a number of unique characteristics and can be a better substitute of other starch sources in a number of food applications, to prepare baby foods, noodles, soups, thickener, extruded products and blending with other ingredients [6]. Due to soft taste, colourless appearance, hypoallergenic properties, low levels of sodium and easy digestible carbohydrates, rice flour is one of the most suitable cereals for glutenfree product formulations [7]. Moreover, it has the smallest particle size and the whitest colour of all commercial grains. The particle size distribution, mean granule size, surface composition, particle shape of rice flour is well known to play an important role in flowability of rice flour.

\section{Flow Property Measurement}

The actual flowability of powder is determined by both the latter's physical properties and the geometry of the system [8].There are some of the experimental methods which can be used for predicting the flow behaviour of rice flour. Generally speaking, a flow of a powder is the relative movement of a bulk of particles among neighbouring particles or along the container wall

\footnotetext{
a Corresponding author: shumailanissar@gmail.com
} 
surface. The forces that are involved are gravitational forces, friction, cohesion (interparticle attraction) and adhesion (particle-wall attraction). A general theory for the determination of flowability of solid particles was developed by Jenike [8].

\subsection{Angle of Repose}

Probably, the simplest test from a technical point of view is the measurement of the angle of repose. It is the angle formed by the horizontal base of the bench surface and the edge of a cone-like pile of granules. It is generally considered that the powders with angle of repose of less than about 40 degrees are free flowing. Powders exhibiting repose angle of 50 degrees and more are likely to cause flow problems. Because the formation of the cone involves frictional forces, interparticle cohesive forces, and impact effects that may lead to segregation, the actual measurements depend on the experimental method procedure. Moreover, the factors that affect the flowability of any given powder are numerous which may also vary the angle of repose includes shape and size distribution.

\subsection{Bulk density and compressibility}

Consumers expect that the mass indicated on the package corresponds to a volume filling the package almost completely that's why the bulk density is important. The bulk density is primarily determined by the solid density and the particles array in the bed. Basically the solid density is an intrinsic property of the material, the array which the particle forms is not. Food powders have irregular particle shapes, and the effects of friction or cohesive forces are significant. Availability of mechanical forces supporting the open structure, such as friction, cohesion, and particle shape determines the stability or existence of a food powder's unconfined arrangement of particles. Therefore, as a result of excessive variability inherent in the system, bulk density alone cannot predict the flowability of powders [9]. Bulk density varies as a function of the applied pressure to it as it is not a single or even a dual value of a given powder. Material's compressibility is represented by a slope or straight line of a log-log plot by measuring the degree to which a powder compacts as a function of the applied pressure. The resulting data provides the information to evaluate wall friction and feeder operation requirements [10].

\subsection{Dynamic flow properties measurement by FT4 Powder Rheometer}

Dynamic flow properties or dynamic characterization of a sample is actually the measurement of a powder in motion. Several techniques for visual characterisation of particle shape have employed optical microscopes, electron microscopy and, more recently, X-ray micro tomography $[2,11]$. But such techniques are either time consuming or labour intensive. Moreover, many other measurement techniques such as Carr's index, Hall flowmeter, shear cell and powder rheometer, also provides powder flow properties $[12,13]$. Though the relationship between particle shape and flow characteristics has not been extensively studied, the samples studied are mostly limited to spherical/ spheroidal particles [14]. The flow properties of three dissimilar materials with similar particle sizes, using permeability and shear cell were analysed by Bumiller [15] and suggests that the particle shape might have significant effects on powder flow properties.

Dynamic flow properties were evaluated by rotating a precision blade through the powder along a fixed helical path. Specific energy (SE) is the energy required to establish a particular flow pattern in a conditioned (conditioning prior to measurement eliminates the effects of inherent powder variability so analysis is both reproducible and repeatable) precise volume of powder. This flow pattern is an upward clockwise motion of the blade, generating gentle lifting and a low stress flow mode in the powder. As it is being established on the upward traverse and the powder is unconfined, the energies measured are more dependent on the forces between particles and less influenced by other factors, such as compressibility. The SE value is calculated from the work done per unit mass in moving the blade through the powder from the bottom of the vessel to the top [16]. Powder flow characteristic measurements clearly showed that differences in particle size distribution and, particularly, particle shape, significantly affect all flow properties over a wide range of stress conditions.

An FT4 Powder Rheometer (Freeman Technology), shown in Fig. 1 is used to measure powder properties such as shear and wall friction, as well as bulk properties like permeability, compressibility, and density. This allows comprehensive characterization of powders from a fluidized state to a highly consolidated one.

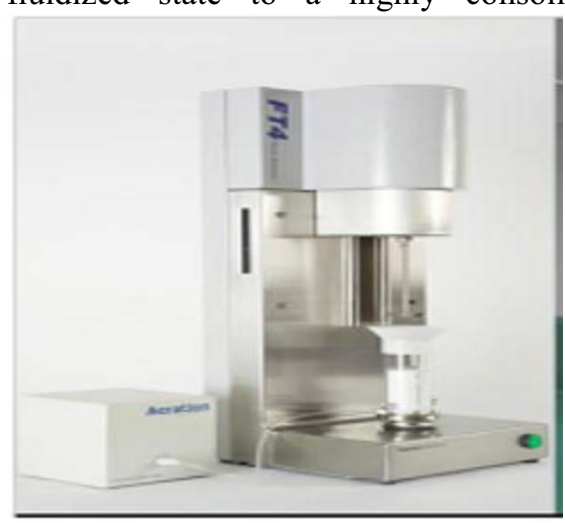


Fig.1. FT4 Powder Rheometer (Freeman Technology)

\subsection{Permeability}

It shows how easily a material can transmit a fluid through its bulk, also using the vented piston attachment, as shown in (Fig.2). The permeability test has a testing sequence similar to that of the compressibility test, though it measures the air pressure required to maintain a constant air flow through the powder bed whilst being consolidated at increasing normal stresses. As the permeability is reduced due to particles becoming more closely packed, a higher normal stress results in a higher pressure drop [16].

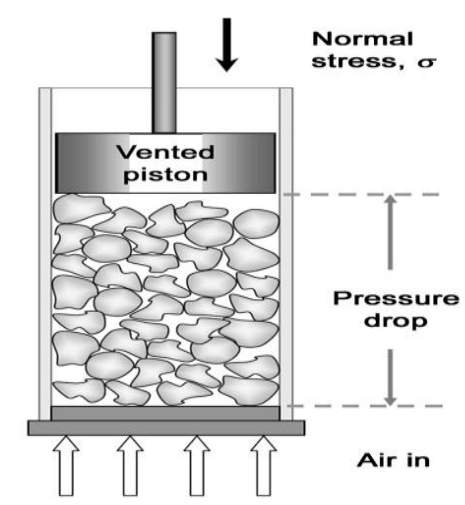

Fig. 2. Material flow through vented piston attachment

\subsection{Shear Test}

The unconfined yield locus and the flow function can be determined by the shear testing. Shear analysis in terms of principal stresses and Mohr's failure criteria is currently the most commonly parameters used for flowability analysis. The main aim of the shear analysis is to provide information about the stress a powder can support before it yields. The most convenient parameter is the unconfined yield stress which generally represents the stress level that can be supported at the compact surface. The unconfined yield stress can be used as an independent measure of powder cohesiveness [17] or be used for the measurement of Jenike's flow function. The latter is the relationship between the unconfined yield stress (or force) and the major consolidation stress (or force) as shown in (Fig. 3), and besides providing data for quantitative flowability classification it can also be used for construction design of silos.

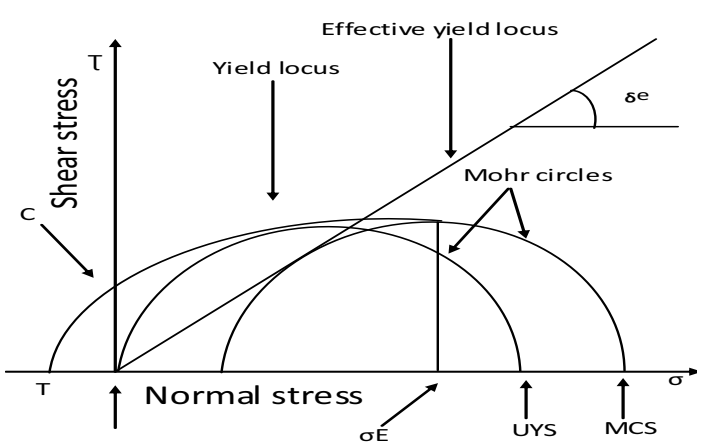

Fig. 3. Relationship between the UYS and MCS (UYS: unconfined yield stress, MCS: major consolidation stress

\section{Conclusion}

Measurement/characterisation of rice flour flowability is useful because it quantifies the strength of the flour that has to be overcome to cause the powder particles to move over each other and flow. This is important in many powder handling and processing operations. In addition, it can be applied for evaluating the effect of processing conditions, such as temperature, relative humidity, and storage time in a silo. Besides these flow measurements, there are numerous methods which can be used for measuring/characterizing the rice flour in terms of flowability as the actual flow depends on both the powder properties and the geometry and other physical characteristics of the system.

\section{References}

1. J.H. Lin, H. Singh, Y.T. Chang, Y.H. Chang, Food Chemistry. 1;126(3):1108-14 (2011)

2. J. Bao, C.J. Bergman, Starch in Food: Structure, Functional and Application. CRC Press, Cambridge, pp. 258-292 (2004)

3. K. Masuda, K. Higashitani, H. Yoshida, Powder Technology Handbook, Third edition CRC Press (2006)

4. M. Peleg, J. Food Proc. Eng. 1:303-28 (1978)

5. C.P. Villareal, B.O. Juliano, S. Hizukuri,. Cereal Chem. 70, 753-758 (1993)

6. B.O. Juliano, Academic Press Inc, New York (1984)

7. H. S. Gujral, I. Guardiola, J. V. Carbonell, \& C.M. Rosell, Food Chemistry, 51, 3814-3818 (2003)

8. A.W. Jenike, Utah Engineering Experiment Station. University of Utah, Salt Lake City (1967)

9. P. Juliano and G.V. Barbosa-Cánovas, Annual review of food science and technology 1:211239 (2010)

10. A.W. Jenike, Bulletin of the University of Utah, 53 and 26 (Bull No. 123 of Utah engineering experiment station, eleventh printing (1986). 
11. X. Fu, M. Dutt, A.C. Bentham, Powder Technology, 167, 134-140 (2006)

12. R.L. Carr, Chemical Engineering 72(3) 69-72. (1965)

13. R.E. Freeman, Powder Technology, 174(1/2), 25-33 (2007).

14. H.Hou, \& C.C. Sun, Journal of Pharmaceutical Sciences, 97(9), 4030-4039 (2008)

15. M. Bumiller, J. Carson, \& J.A. Prescott, In World congress on particle technology 4 Sydney, Australia (2002)

16. F. Xiaowei, H. Deborah, M. Lisa, B. Armstrong, U. Willen, T. Freeman, Particuology 10, 203- 208 (2012)

17. P. York, Powder Technology 11, 197 (1975). 